\title{
MONITORING OF IMPLANT STABILITY WHEN PLACED SIMULTANEOUSLY IN PLATELET RICH FIBRIN ENHANCED MAXILLARY SINUS BONE AUGMENTATION
}

\author{
Mohammed Ahmed Elsholkamy* and Gihan Eldesouky **
}

\begin{abstract}
Background: Restoring posterior maxilla with implants is challenging due to lack of bone quantity and quality.

Objective: Assess the effect of adding platelet rich fibrin (PRF) to bovine bone mineral (Bio oss) as sinus graft with simultaneous implant placement.

Patients and methods: Sixteen implants were placed in 16 augmented sinuses divided into two groups; the first (study) group sinus augmentation was done using Bio oss and PRF and the second (control) group using Bio oss alone. Implant stability and bone density were assessed.

Results: The sinuses grafted with Bio oss mixed with PRF showed significantly higher bone density and implant stability after 6 months.

Conclusion: The use of Bio oss mixed with PRF as a graft materiel in sinus lifting procedure is highly superior to Bio oss alone with respect to the implant stability and bone density around the implant.
\end{abstract}

KEY WORDS: PRF, sinus augmentation, implant stability, Osstell ISQ.

\section{INTRODUCTION}

Implant dentistry is an every-day practice and a common treatment modality to replace missing teeth and rehabilitate both completely and partially edentulous cases. Since Branemark introduced his revolutionary and contemporary concept of osseointegration, the dental implant has become an integral component of modern dental practice. ${ }^{(1)}$ The ambitions, expectations, and success criteria have shown a great shift from just mere osseointegrated implants to full esthetic rehabilitation including soft tissue management and bone augmentation.

\footnotetext{
* Assistant Professor of Oral \& Maxillofacial Surgery, Faculty of Dentistry, Suez Canal University.
}

** Professor and Chairman of Oral Radiology, Faculty of Dentistry, Suez Canal University. 
The ideal candidate for dental implant procedure must have sufficient bone volume in both maxillary and mandibular ridges to accommodate these implants. ${ }^{2}$ Good bone quality also is an important ruling factor in the implant stability and future success. ${ }^{3}$ Unfortunately, many cases present as complicated or even deteriorated. It is obviously known that natural resorption occurs after extraction that causes deficiency in bone volume, thus augmentation of the alveolar ridge before implant placement will be needed. ${ }^{4}$ Moreover, the posterior maxilla has some anatomic limitations such as flat palatal vault, inadequate posterior alveolar width, and deficient alveolar height. In general, maxillary bone is mostly trabecular with less bone density and quantity than the premaxilla or mandible. Adjacent cortices of compact bone are generally very thin, providing minimal strength. ${ }^{2}$

In the immediate period following maxillary posterior teeth extraction, initial decrease in alveolar width takes place due to resorption and/or loss of buccal bone. Furthermore, continuous bone remodeling, absence of periodontal ligament and proprioceptive stimulation, loss of bone height and density along with increase in antral pneumatization lead to more deterioration of the residual bone condition. The maxillary sinus pneumatization is due to progressive hollowing out of alveolar process from the apical aspect mediated by osteoclasts and increase in positive intra-antral pressure. In such a situation, the residual vertical bone height is decreased making standard implant placement difficult. ${ }^{5,6}$

In the past 3 decades, implant dentistry has passed through development and innovation in adjunctive surgical techniques, biomaterials, and grafting materials and techniques to facilitate implants placement in complicated situations. In the third Millennium, a well-trained operator can replace teeth defects usually in most instances for an adult individual who is willing to cooperate, comply with the treatment plan details, and to provide the required financial contribution. Owing to such development in surgical techniques, the contraindications for implants due to bone volume and density deficiency have become little. ${ }^{5}$

The sinus lift and augmentation is among the most important surgical approaches to help implant insertion in the posterior maxilla. There are two main surgical approaches to augment the sinus and since their introduction, they have been predictable as well as reproducible. The Tatum lateral sinus approach using a Caldwell-Luc osteotomy is historically the first main technique and has been developed in the mid-1970s. ${ }^{7,8}$ The axial or crestal approach via the implant osteotomy itself was first developed by Tatum to simplify the procedure without the surgical opening of the sinus cavity. ${ }^{9,10} \mathrm{His}$ objective was to use the natural osteogenic capabilities of the Schneiderian membrane to gain the missing few millimeters of bone around the implant tip. Misch first published it in $1987^{11}$ but it became more famous after Summers published a similar technique in 1994 and since then it became popular as "Summers Osteotomy". ${ }^{12}$ This approach also helps to reduce the grafting volume to the minimum. The height of the residual bone between the floor of the sinus and the alveolar crest is the key determinant factor that dictates the choice of the sinus augmentation approach, either the lateral via Caldwell- Luc osteotomy or axial via Summers osteotomy. 8,13,14,15

Some of the sinus lift approaches involve simple minimal elevation of the Schneiderian membrane, ${ }^{16}$ while others include placement of various types of grafts. Many graft materials are used to augment the maxillary sinus like autogenous bone, allografts, xenografts, bone morphogenetic proteins, and alloplastic materials as $\beta$-tricalcium phosphate or hydroxyapatite. There is consensual belief that most of the used materials are efficient for such surgery, bearing in mind the high osteogenic potential of the Schneiderian membrane being 
having a periosteum-like behavior. However, the choice of certain graft material or combination of several ones will influence the waiting period before optimum healing and remodeling of the grafted area and hence future implant placement and / or functional loading. ${ }^{17,18}$

Choukroun et al in France first described Choukroun's platelet-rich fibrin (PRF) in 2001. ${ }^{19}$ This fibrin clot autologous biomaterial releases large amounts of growth factors in vitro during, at least, seven days such as platelet-derived growth factor, vascular endothelial growth factor, and transforming growth factor $\beta 1 .^{20} \mathrm{PRF}$ applications were described in oral and maxillofacial surgery, preimplant and implant surgery. ${ }^{21-25}$ Moreover, PRF proved efficacy when employed in sinus augmentation for both lateral and osteotome sinus lift approaches. ${ }^{26,27}$

Implant stability in the alveolar bone comprises two main entities, which interlace with each other. The initial primary stability at the time of implant insertion and the secondary long-term stability. In general, it is a crucial issue for the implant prognosis and success. Good primary implant stability is a key factor for successful immediate loading. It favors the osseointegration since it limits micro-movements at the bone-implant interface. Accordingly, most studies recommended that poor primary stability is an exclusion factor for immediate loading. ${ }^{28,29}$ Several methods had been used to assess implant stability however, the most popular of which are the reverse-torque or removal torque test, the periotest, and resonance frequency analysis (Osstell method). 29,30 The current study aimed to evaluate the effect of adding PRF to the sinus graft material along with simultaneous implant insertion on the implant stability, bone quality, and the overall outcome.

\section{PATIENTS AND METHODS}

This study took place at the outpatient clinic of oral and maxillofacial surgery department, Faculty of dentistry, Suez Canal University.

\section{Patients sampling and study design:}

This case series comprised placing sixteen implants in grafted sinuses after Schneiderian membrane elevation using buccal lateral (CaldwellLuc) approach.

The main inclusion factor was to recruit patients with missed maxillary posterior teeth for long time with pneumatization of the maxillary sinus so that they have crestal bone height of minimum $5 \mathrm{~mm}$, which was insufficient for routine implant placement. This criterion followed the Misch subantral (SA) classification and coincided with his subantral (SA) option number 3 that would allow simultaneous implant placement with good initial stability depending on the residual minimum bone height. ${ }^{8}$

Exclusion criteria included smoking patients, patients with immunologic diseases, uncontrolled diabetes mellitus, and patients that had chemotherapy or radiotherapy or long term intravenous bisphosphonates intake. Other contraindicating systemic conditions affecting bone healing were also excluded.

The Sixteen implants were divided randomly and equally into two groups:

Group one (study group): The sinus augmentation was done using a mixture of bovine bone xenograft and PRF.

Group two (control group): The sinus augmentation was done using bovine bone xenograft alone.

\section{Preoperative assessment:}

All patients had read and signed an informed consent concerning their approval to be enrolled in the study protocol. The consent explained the study methodology briefly pointing out the personal and societal benefits as well as explaining the alternatives and potential hazards. Digital 
panoramic radiographs (OPG) were performed for the patients preoperatively to detect if the patient needed sinus lift procedure and assess provisionally that the residual bone height conformed to our inclusion criteria. It was also beneficial to rule out any central lesions or pathology involving the sinus. The neighboring teeth condition and their relation to the edentulous site as well as any root curvatures or abnormality that might affect the treatment plan was assessed. Cone Beam computerized tomography (CBCT) (Scanora 3D, Soredex, Tuusulu, Finland) was performed to examine the area of interest. We measured bone width and height making sure that the selection of the implant size was suitable for the residual bone and that the patients had a minimum residual height of bone at the edentulous area conforming to the Misch SA option number 3 (Fig. 1, A and B). Clinical assessment and study models were done to assess the interarch space, the mesiodistal space, the width of the ridge, and the mucosal biotype.

\section{Operative procedures}

All patients had read and signed an informed consent declaring their approval to undergo the surgical procedures. The consent explained in detail the surgical procedures and their potential hazards as well as the alternatives to such surgery. The same surgeon, using standardized techniques under aseptic conditions, performed all the surgical procedures. All surgical procedures were carried out under local anesthesia using Articaine hydrochloride 4\% with 1:100.000 epinephrine (Ubistesin forte, 3M ESPE Germany). Anesthesia was achieved by buccal infiltration technique for the buccal mucoperiostium, maxillary sinus, and alveolar bone and palatal infiltration technique for the palatal mucosa.

\section{Preparation of Platelet-Rich Fibrin}

PRF was prepared twenty minutes before starting surgery, $10 \mathrm{~mL}$ of venous blood was collected in a sterile dry, neutral glass tube without an anticoagulant. Centrifugation was performed immediately at (3000 rpm) for 10 minutes. The platelet-poor plasma, which accumulated at the top of the glass tube was discarded. PRF was dissected approximately $2 \mathrm{~mm}$ below its connection to the red corpuscle layer beneath to include remaining platelets, which have been proposed to localize below the junction between PRF and the red corpuscle (Fig. 2, A, and B). ${ }^{19}$

\section{Surgical procedures}

Incisions were performed and a standard threeincision line pyramidal flap (trapezoid flap) was reflected with a mucoperiosteal elevator. A surgical ruler was used to detect the outline of the buccal

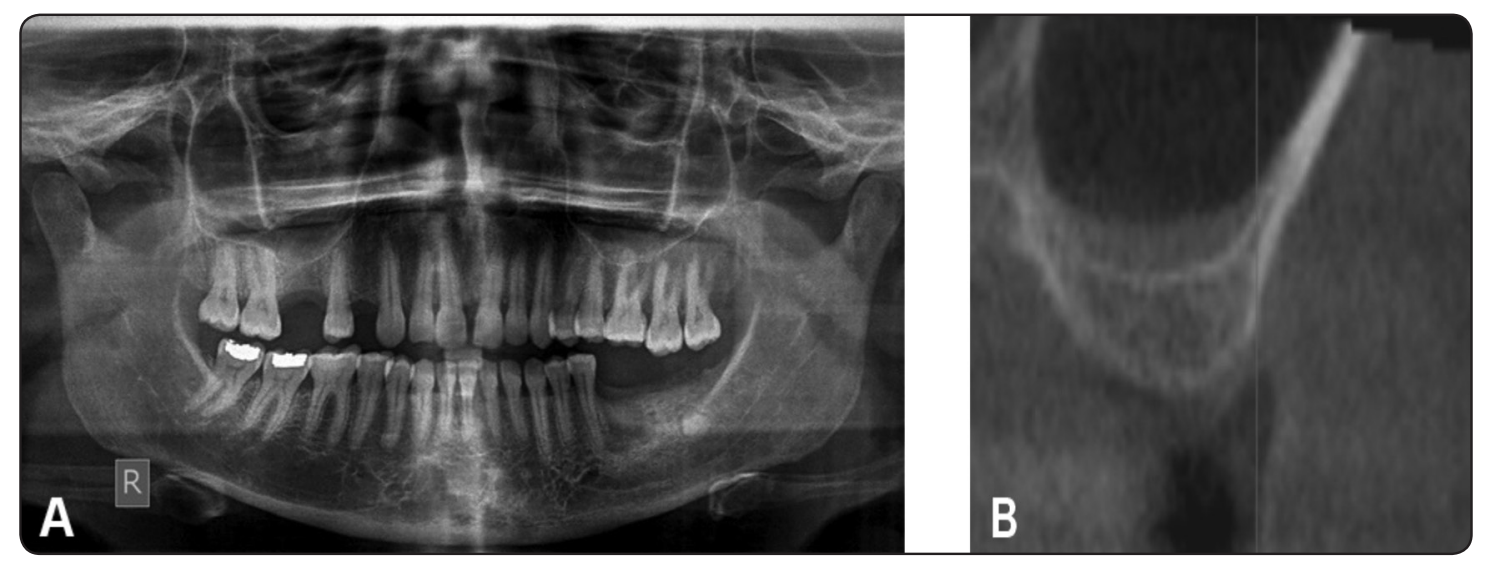

Fig. 1. A, Photograph showing preoperative digital panoramic radiograph (OPG) B, Photograph showing para-axial cut of preoperative $\mathrm{CBCT}$ to detect bone width and height at the area of interest. 
window and its position from the alveolar crest. A large round diamond bur mounted on a low speed high torque straight hand piece was used to create the buccal window under copious saline irrigation. Sinus membrane was elevated cautiously using Ace sinus elevators (Ace surgical supply Co. USA). Implant preparation osteotomy was performed according to the manufacturer directions. Platelet rich fibrin, prepared prior to the surgery, was mixed with bovine bone xenograft (Bio oss, Geistlich Pharma AG, Wolhusen Switzerland) (Fig. 3, A and B). In the study group cases, this mixture was gently packed as sinus grafting material inside the previously elevated sinus cavity, right next to the elevated membrane on the medial side of the proposed implant position. Osstem implant (Osstem
Implant Co., Ltd. South Korea) with suitable diameter and length according to residual bone was then placed in the desired previously prepared position (Fig. 4). Afterwards, the graft material was gently packed on the lateral aspect of the implant to fill the whole osteotomy window. In the control group, bovine xenograft (Bio oss) was packed alone in the sinus and then the packing pattern and implant placement continued entirely similar to that of the study group.

Two cases in the study group had an accidental minor tear in the Schneiderian membrane during elevation and was dealt with promptly. A portion of the prepared PRF was compressed between two sterile glass slabs and used as a membrane to cover

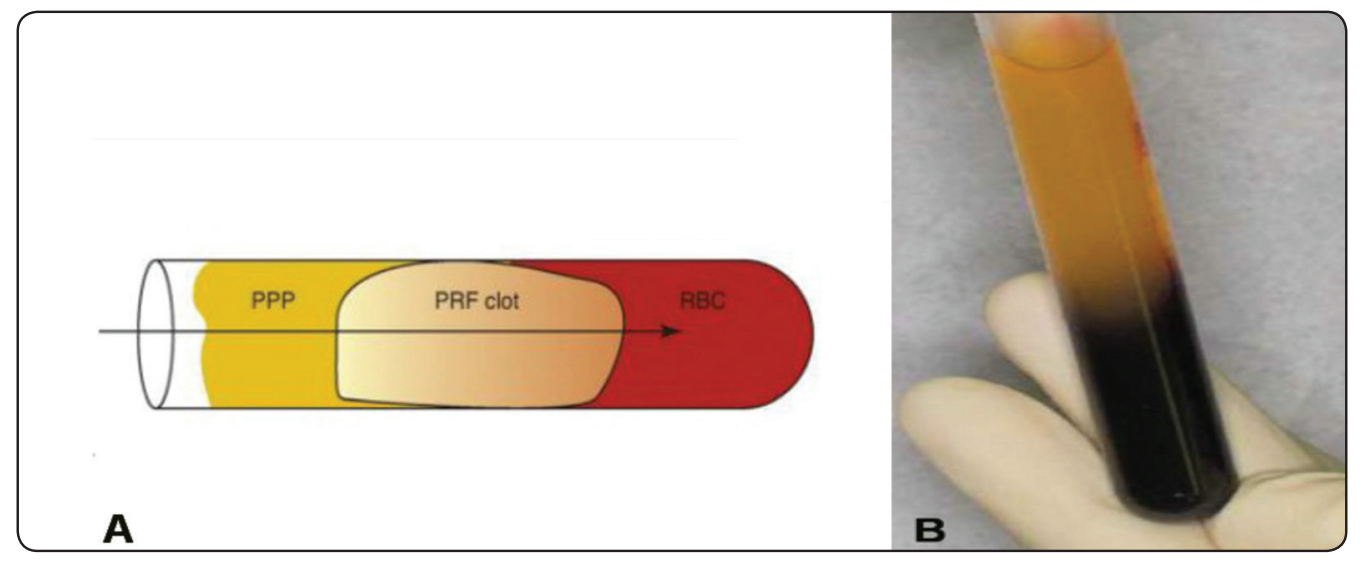

Fig. 2. A, A diagram showing the three layers after centrifugation with the PRF layer in the middle and a poor platelet plasma (PPP) above and red corpuscles beneath. B, A clinical photograph showing the glass tube with the PRF layer in before its dissection from the red cell corpuscles beneath.

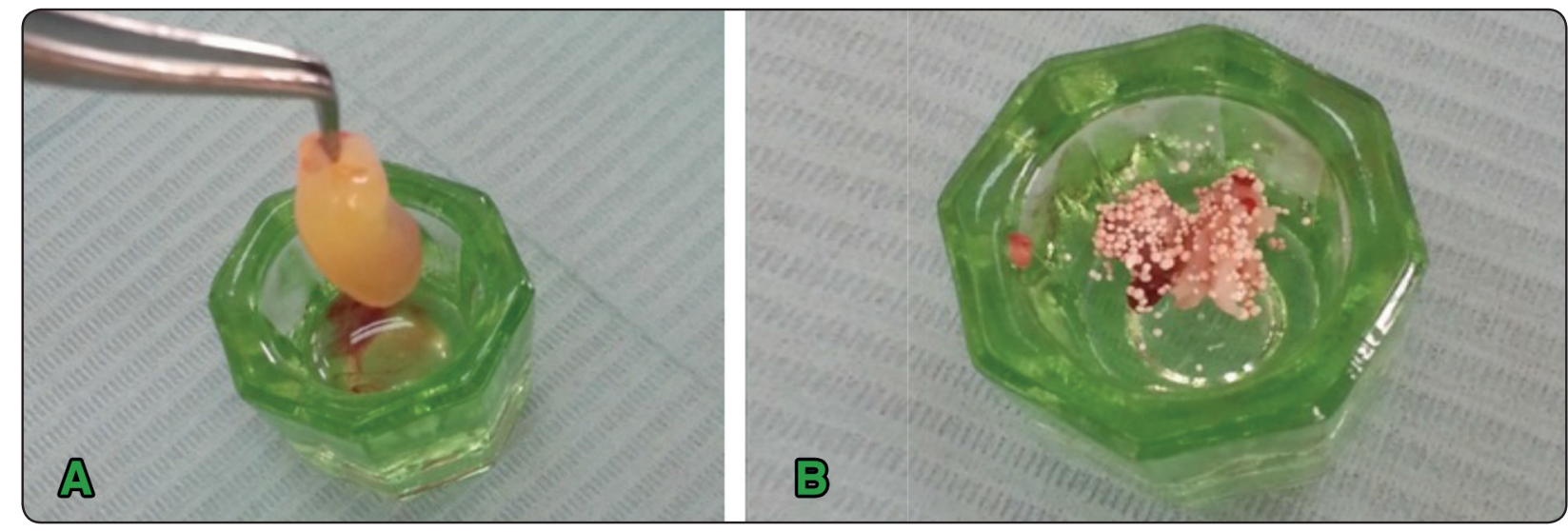

Fig. 3. A, Clinical photograph showing PRF after separation kept in a sterile glass container. B, A clinical photograph showing bovine bone graft mixed with PRF. 
the membrane tear site. One case in the control group had the same membrane tear while proceeding with the elevation. We used an absorbable lyophilized collagen membrane (Biocollagen, Bioteck, Vicenza, Italy) to cover the tear and allow packing of bone graft. The wounds were closed with absorbable polyglycolic acid suture (Egysorb, Taisier-med, Egypt). The patients were asked to keep biting on a sterile gauze pack for one hour postoperatively.

The appropriate postoperative instructions regarding the postoperative sequelae as pain and swelling was explained to the patients and given also in printed form to ensure their compliance and safety. The sinus protection instructions to prevent any changes in the intra-antral pressure by avoiding whistling, blowing, using a straw, sneezing and/or nose blowing were all explained to the patients and printed also as well.

The appropriate postoperative drugs were prescribed for all patients. The antibiotic,Amoxicillin trihydrate $875 \mathrm{mg}$ + Clavulanate potassium $125 \mathrm{mg}$ (Augmentin 1gm, Medical Union Pharmaceuticals, MUP, Egypt for GlaxoSmithKline pharmaceuticals Ltd., GSK, London, UK) was recommended twice daily orally for one week. The analgesic and antiinflammatory Ibuprofen (Brufen 400 mg, El Kahira/ ABBOTT, Egypt) was prescribed three times a day

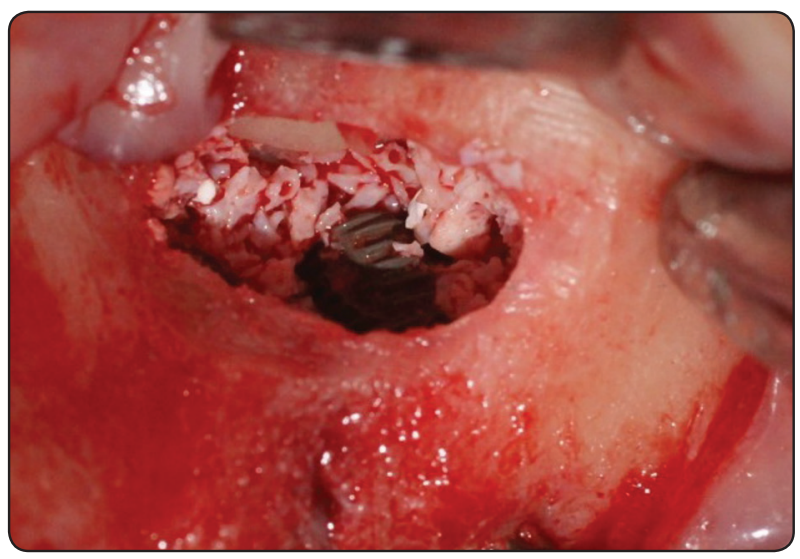

Fig. (4) A clinical photograph showing the implant in position extending in the sinus cavity surrounded by a mixture of PRF and bovine bone (Bio oss). orally for 5 days. Alpha Chymotrypsin $5 \mathrm{mg}$ (Amoun Pharmaceutical Co., Egypt/ Leurquin, France) was prescribed once daily for three days. Xylometazoline Hydrochloride $0.1 \%$ (Otrivine Adult Nasal Drops $0.1 \%$, GlaxoSmithKline Consumer Healthcare, UK) was prescribed three times daily for 3-5 days.

\section{Postoperative assessment:}

Digital panoramic radiograph was taken for each patient one day postoperatively to assess the surgical procedure (Fig. 5). Each patient returned for clinical follow up once weekly for three weeks postoperatively. CBCT was used for assessment of bone density around the implants after 4 and 6 months postoperatively and the $\mathrm{CBCT}$ software was used to detect the bone density around the imlpant (Figs. 6-9). The implant stability was assessed using Osstell ISQ device (Integration Diagnostics Ltd. Company, Sävedalen, Sweden) immediately after implant insertion, after 4 months, and 6 months postoperatively. The abutment placement decision was based on the Osstell readings. When the reading was 70 or more, the abutment was placed and proceeding with finishing the temporary or the final prosthesis was carried out (Figs. 10-13).

The data collected from the bone density measurements and the Osstell readings as well as

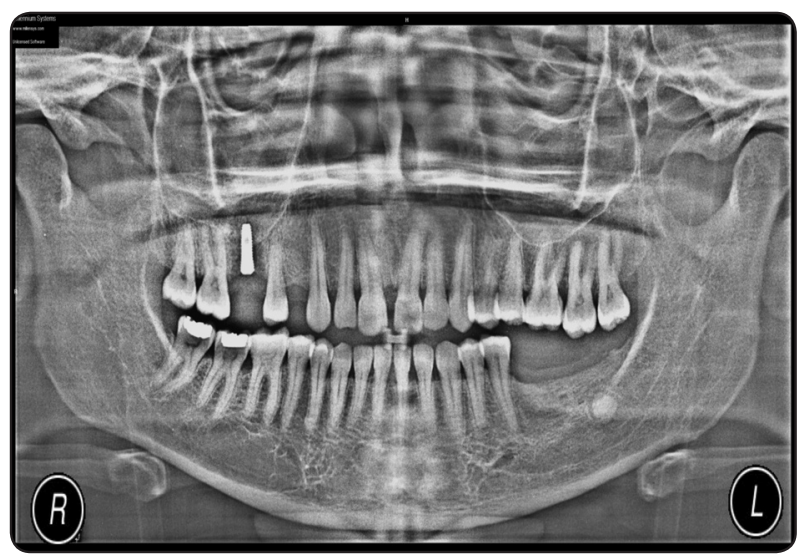

Fig. (5) Photograph showing postoperative digital panoramic radiograph revealing the implant in good alignment inside the grafted sinus. 


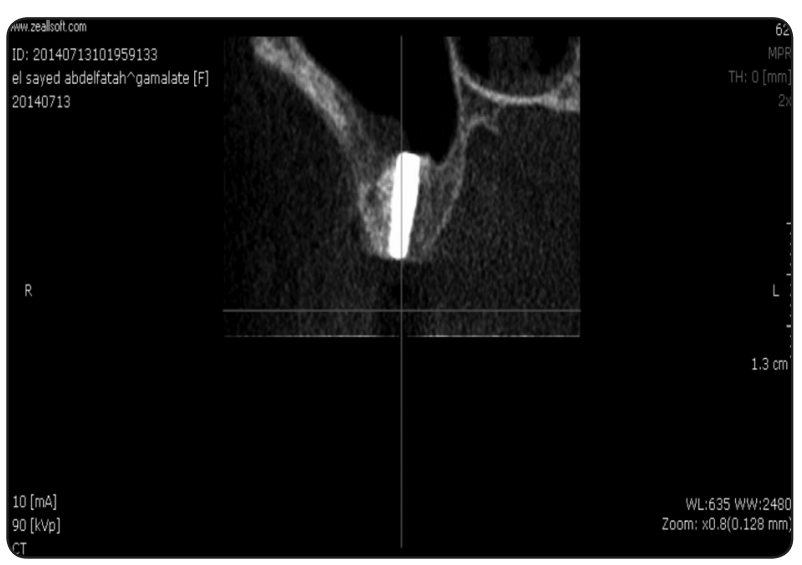

Fig. (6) Photograph of para-axial CBCT cut at 4 months postoperatively showing bone graft healing around the implant.

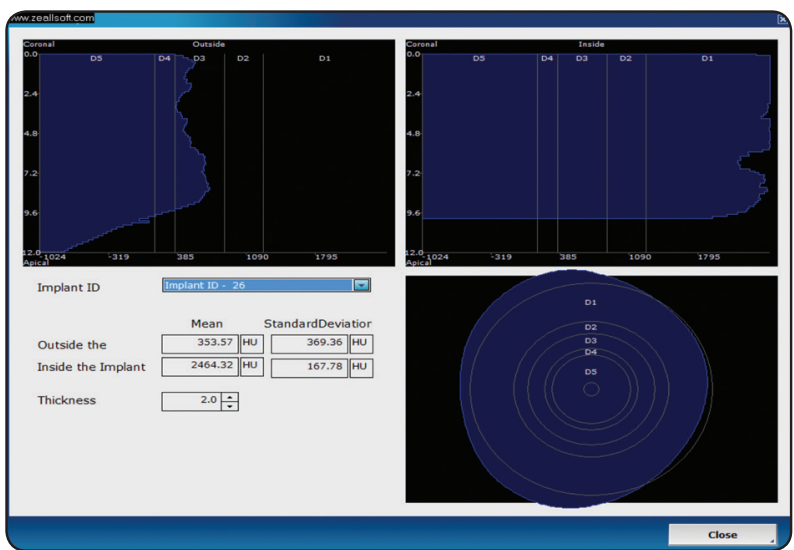

Fig. (8) Photograph showing CBCT software application to detect bone density around the implant in the newly formed bone.

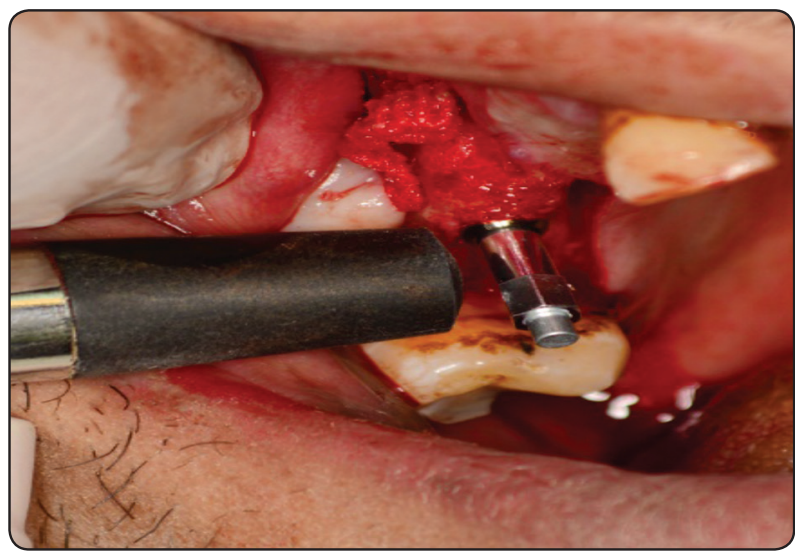

Fig. (10) Clinical photograph showing the smart peg in place, attached to the implant, and the Osstell ISQ device probe placed near to it to assess the implant stability.

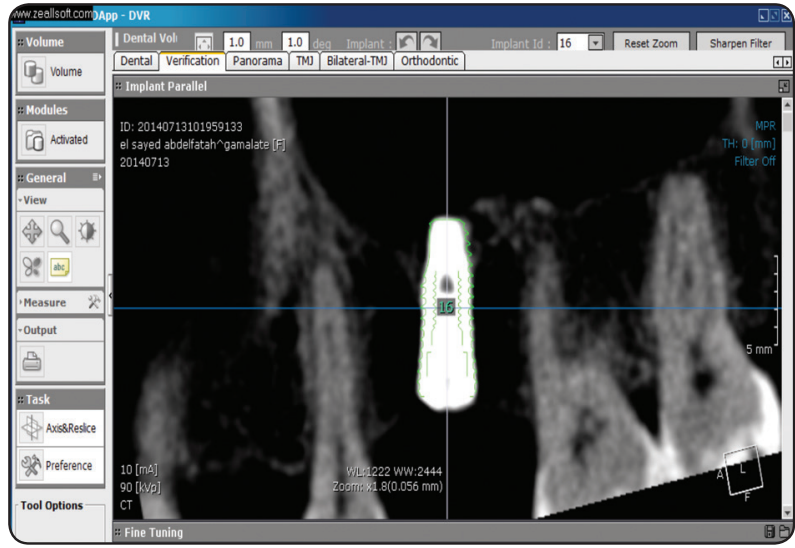

Fig. (7) Photograph showing CBCT software to detect bone density

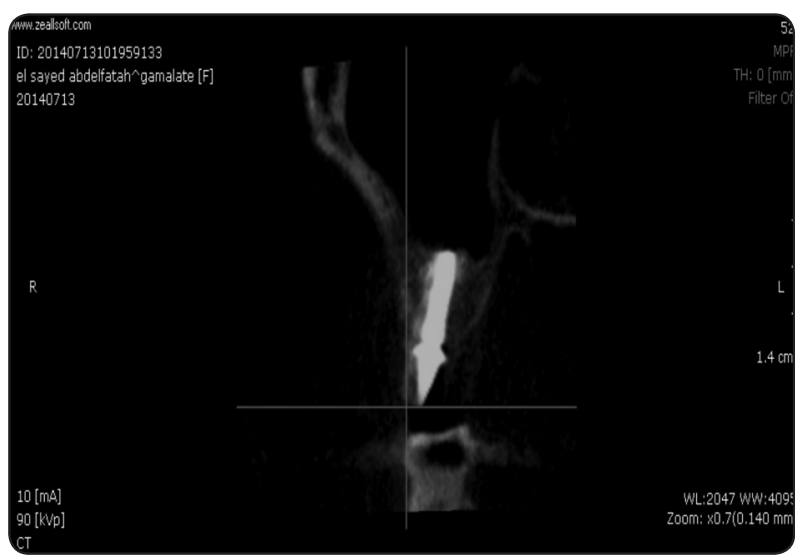

Fig. (9) Photograph of para-axial cut CBCT at 6 months postoperatively showing bone around the implant filling the augmented sinus and the abutment attached to the implant.

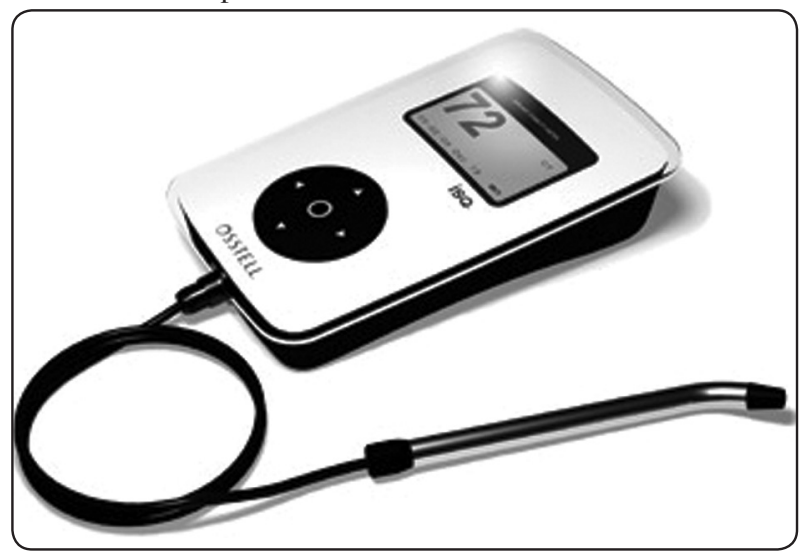

Fig. (11) Clinical photograph showing Osstell ISQ device reading for a case from the study group. 


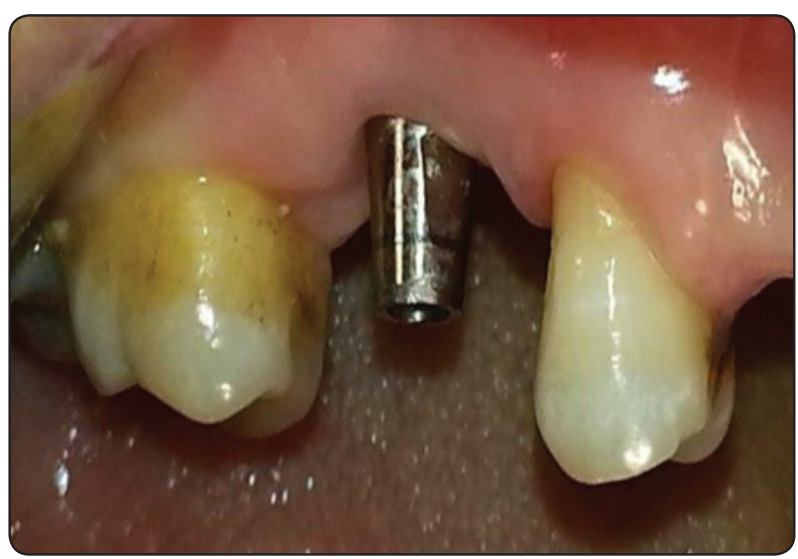

Fig. (12) Clinical photograph showing the abutment attached to the implant with surrounding good gingival healing.

the clinical and personal data was saved, tabulated, and statistically tested.

\section{RESULTS}

Sixteen implants were inserted in 16 patients in the current study to detect the efficacy of Platelet Rich Fibrin (PRF) when mixed with the graft material during sinus lift with simultaneous implant placement. The cases were divided randomly and equally into two groups; a study group (that received PRF mixed with bovine bone) and a control group (that received bovine bone only).

The mean age of the cases among the study group was 40.3 years old and that among the control group was 40.1 years with an overall mean of 40.2 years for the entire sample. Regarding the gender, $37.5 \%$ of the cases among the study group were males while $62.5 \%$ were females, whereas, among the control group, males and females were equally distributed. The majority of the cases were females with a percentage of $56.3 \%$ (Table 1). The differences between the research groups in both age and gender were not statistically significant.

The clinical assessment showed almost uneventful healing for most of the cases. The edema subsided after three days for all cases except for

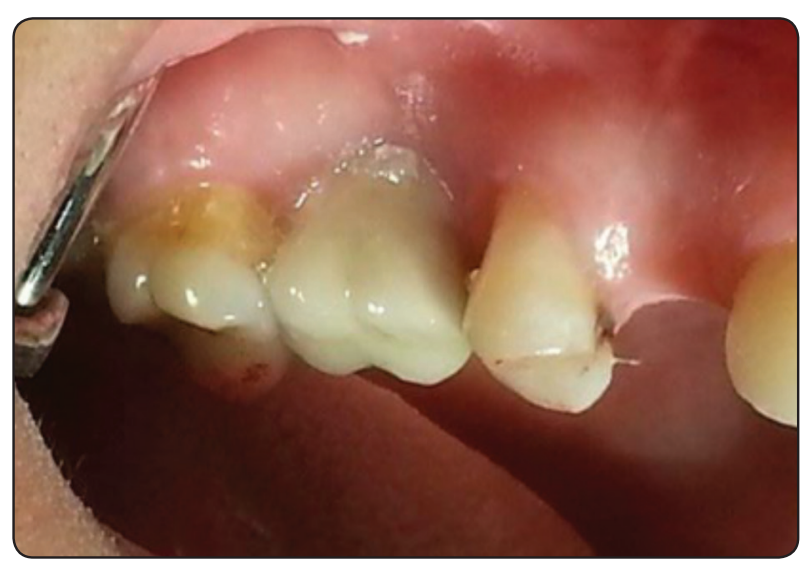

Fig. (13) Clinical photograph showing porcelain fused to metal final prosethsis after cementation.

two. Those were from the control group and had edema that lasted for five days. The cases that had accidental tear in the Schneiderian membrane had no postoperative complications during the various follow up sessions. Wound dehiscence was observed in two cases only in the control group and they had a secondary healing closure later at the third week postoperatively with meticulous care for the oral hygiene.

The values of mean bone density among the study group when measured at 4 and 6 months postoperatively were $311.2 \mathrm{HU}$ and $553.1 \mathrm{HU}$ respectively. Moreover, the increase in the density values was statistically significant (Table 2)

The values of mean bone density among the control group when measured at 4 and 6 months postoperatively were $319.9 \mathrm{HU}$ and $398.8 \mathrm{HU}$ respectively. However, the difference in the density values was not statistically significant (Table 3)

On comparing both groups, the difference between the bone density values at 4 months postoperatively was in favor of the control group however, this was not statistically significant. On the other hand, the mean bone density of the study group was much higher than that of the control group at 6 months postoperatively. Moreover, that 
TABLE (1): Frequency distribution of the studied subjects according to their personal data $(\mathrm{N}=16)$ :

\begin{tabular}{|c|c|c|c|c|c|c|c|c|}
\hline \multirow{2}{*}{\multicolumn{2}{|c|}{$\begin{array}{c}\text { Variable } \\
\text { No. }\end{array}$}} & \multicolumn{2}{|c|}{ Study group $(n=8)$} & \multicolumn{2}{|c|}{ Control group $(n=8)$} & \multicolumn{2}{|c|}{ TOTAL } & \multirow{2}{*}{ P-value } \\
\hline & & $\%$ & No. & $\%$ & No. & $\%$ & & \\
\hline \multirow{2}{*}{ Age } & Mean \pm SD & \multicolumn{2}{|c|}{$40.38 \pm 3.6$} & \multicolumn{2}{|c|}{$40.13 \pm 2.9$} & \multicolumn{2}{|c|}{$40.25 \pm 3.2$} & \multirow{2}{*}{$0.672^{(*)}$} \\
\hline & Min - Max & \multicolumn{2}{|c|}{$35-46$} & \multicolumn{2}{|c|}{$36-45$} & \multicolumn{2}{|c|}{$35-46$} & \\
\hline \multirow{2}{*}{ Gender } & Male & 3 & $37.5 \%$ & 4 & $50 \%$ & 7 & $43.8 \%$ & \multirow{2}{*}{$0.614^{(*)}$} \\
\hline & Female & 5 & $62.5 \%$ & 4 & $50 \%$ & 9 & $56.3 \%$ & \\
\hline
\end{tabular}

(*) Not statistically significant, using Chi square t-test.

TABLE (2): Frequency distribution of the studied subjects among the study group according to the change in the mean bone density $(\mathrm{N}=8)$

\begin{tabular}{|c|c|c|c|c|}
\hline \multicolumn{2}{|c|}{ Variable } & 4 month measurement & 6 month measurement & P-value \\
\hline \multirow{2}{*}{ Measurement } & Mean + SD & $311.2 \pm 39.56$ & $553.1 \pm 100.1$ & \multirow{2}{*}{$\mathbf{0 . 0 0 1}^{(*)}$} \\
\cline { 2 - 5 } & Min - Max & $260.77-372.73$ & $403.72-671.3$ & \\
\hline
\end{tabular}

(*) Statistically significant, using ANOVA test.

TABLE (3): Frequency distribution of the studied subjects among the Control group according to the change in the mean bone density $(\mathrm{N}=8)$ :

\begin{tabular}{|l|c|c|c|c|}
\hline \multicolumn{2}{|c|}{ Variable } & 4 month measurement & 6 month measurement & P-value \\
\hline \multirow{3}{*}{ Measurement } & Mean + SD & $319.9 \pm 68.2$ & $398.8 \pm 64$ & \multirow{2}{\text{0.702}^{(*)}}{} \\
\cline { 2 - 5 } & Min - Max & $238.47-430.16$ & $324.61-498.31$ & \\
\hline
\end{tabular}

(*)Not statistically significant, using ANOVA test.

TABLE (4): Frequency distribution of the studied subjects between both groups according to the change in the mean bone density at the different stages of assessment $(\mathrm{N}=8)$ :

\begin{tabular}{|c|c|c|c|c|}
\hline \multicolumn{2}{|c|}{ Variable } & Study group & Control group & P-value \\
\hline \multirow{3}{*}{4 months measurement } & Mean + SD & $311.2 \pm 39.56$ & $319.9 \pm 68.2$ & \multirow{2}{*}{$\mathbf{0 . 3 3 2}^{(*)}$} \\
\cline { 2 - 4 } & Min - Max & $260.77-372.73$ & $403.72-671.3$ & \\
\hline \multirow{2}{*}{ 6 months measurement } & Mean + SD & $553.1 \pm 100.1$ & $398.8 \pm 64$ & \multirow{2}{*}{$\mathbf{0 . 0 4}^{(* *)}$} \\
\cline { 2 - 4 } & Min - Max & $403.72-671.3$ & $324.61-498.3$ & \\
\hline
\end{tabular}

(*)Not statistically significant, using ANOVA test. $\quad$ (**) Statistically significant, using ANOVA test. 


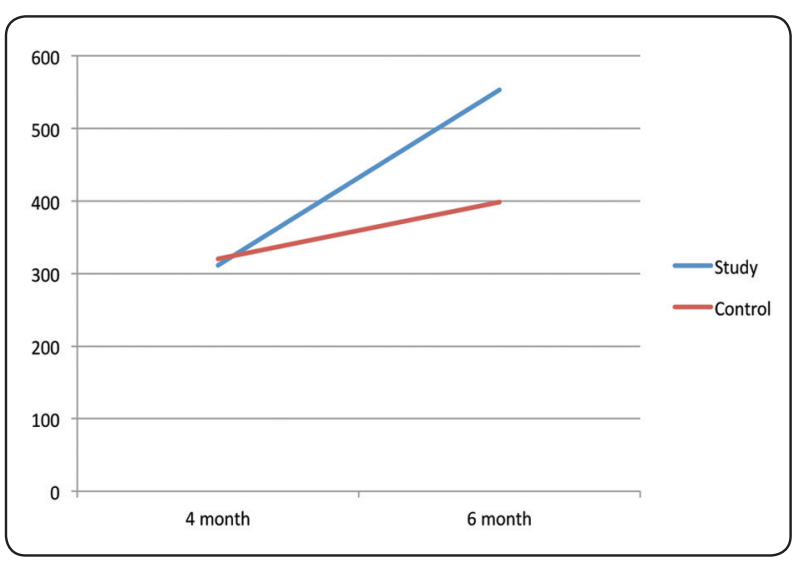

Fig. (14) Graphical representation of the change in bone density in both groups throughout the postoperative assessment sessions.

difference was found to be statistically significant (Table 4. Fig. 14).

The mean implant stability quotient (ISQ) among the study group was measured immediately after implant insertion, after 4 months, and 6 months postoperatively. The values were 65,70 , and 74 re- spectively. After 6 months, all implants reached the desired value for loading (70 ISQ) and were loaded. The increase in the ISQ was significant (Table 5).

The mean implant stability quotient (ISQ) among the control group was measured immediately after implant insertion, after 4 months, and then 6 months postoperatively. The values were 64,66 , and 67 respectively. No implant reached the loading eligible value so the loading was delayed. The difference between values at various assessment sessions was not found statistically significant. (Table 6).

On comparing the ISQ values between both groups, the difference between the mean ISQ immediately after implant insertion was not statistically significant. Likewise, the difference between both groups at 4 months postoperative assessment was not significant also although the study group showed higher mean value. However, at the 6 months postoperative assessment session

TABLE (5): Frequency distribution of the studied subjects among the study group according to the change in the mean implant stability quotient (ISQ) $(\mathrm{N}=8)$ :

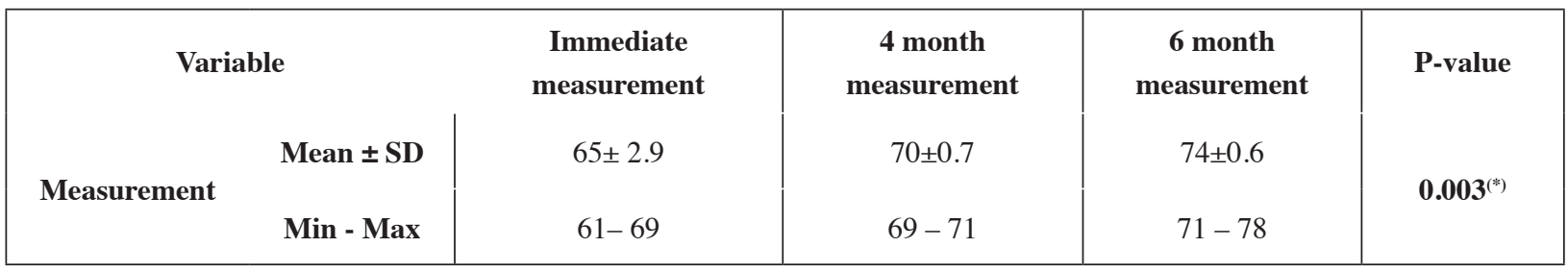

(*)Statistically significant, using Chi square t-test.

TABLE (6): Frequency distribution of the studied subjects among the control group according to the change in the mean implant stability quotient (ISQ) $(\mathrm{N}=8)$ :

\begin{tabular}{|c|c|c|c|c|c|}
\hline \multicolumn{2}{|c|}{ Variable } & $\begin{array}{c}\text { Immediate } \\
\text { measurement }\end{array}$ & $\begin{array}{c}\text { 4 month } \\
\text { measurement }\end{array}$ & $\begin{array}{c}\text { 6 month } \\
\text { measurement }\end{array}$ & P-value \\
\hline \multirow{2}{*}{ Measurement } & Mean + SD & $64 \pm 4.1$ & $66 \pm 3.1$ & $67 \pm 2.2$ & \multirow{2}{*}{$\mathbf{0 . 3 0 2}(*)$} \\
\cline { 2 - 6 } & Min - Max & $56-68$ & $60-69$ & $63-69$ & \\
\hline
\end{tabular}

(*)Not statistically significant, using Chi square t-test. 
TABLE (7): Frequency distribution of the studied subjects between Both groups according to the change in the mean implant stability quotient (ISQ) at the different stages of assessment ( $\mathrm{N}=8$ ):

\begin{tabular}{|c|c|c|c|c|}
\hline \multicolumn{2}{|c|}{ Variable } & Study group & Control group & P-value \\
\hline \multirow{2}{*}{ Immediate } & Mean \pm SD & $65 \pm 2.9$ & $64 \pm 4.1$ & \multirow{2}{*}{$0.402^{(*)}$} \\
\hline & Min - Max & $61-69$ & $56-68$ & \\
\hline \multirow{2}{*}{4 months measurement } & Mean \pm SD & $70 \pm 0.7$ & $66 \pm 3.1$ & \multirow{2}{*}{$0.261^{(*)}$} \\
\hline & Min - Max & $69-71$ & $60-69$ & \\
\hline \multirow{2}{*}{6 months measurement } & Mean \pm SD & $74 \pm 0.6$ & $67 \pm 2.2$ & \multirow{2}{*}{$\mathbf{0 . 0 0 3}^{(* *)}$} \\
\hline & Min - Max & $71-78$ & $63-69$ & \\
\hline
\end{tabular}

(*)Not statistically significant, using ANOVA test. $\quad$ (**) Statistically significant, using ANOVA test.

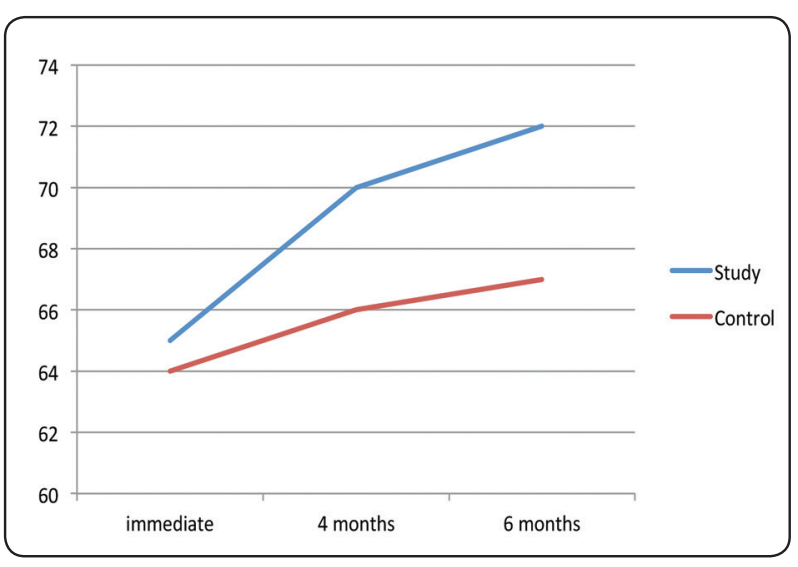

Fig. (15) Graphical representation of the increase in mean implant stability quotient (ISQ) among both the study and the control groups throughout the whole study.

the study group also showed higher ISQ mean value and the difference between both groups was found statistically significant (Table 7. Fig. 15).

\section{DISCUSSION}

The current study aimed to evaluate the effect of adding PRF to the sinus graft material on the osseointegration outcome as assessed via implant stability (ISQ value) and bone density. Sixteen implants were inserted in a $5 \mathrm{~mm}$ minimum height of bone simultaneously with sinus augmentation fulfilling the
Misch SA option number $3 .{ }^{8}$ The rationale for such choice was to minimize the healing and rehabilitation time as the implants would osseointegrate with the graft maturation. The presence of the implants projecting into the sinus cavity helps to keep the Schneiderian membrane up to an adequate height acting as tent pegs. This supports the graft material holding enough room for the newly formed bone to occupy.$^{31}$ Besides, the implant osteotomy performed in the residual bone at the sinus floor elicits regional acceleratory phenomenon that involves intense localized bone modeling and remodeling to help postoperative healing. ${ }^{32,33}$

We used the CBCT in the present study to detect the bone density around the implants after 4 and 6 months postoperatively. OPG was used immediately after the procedure to check the position and the angulation of the implant and the overall outcome of the sinus augmentation. We did not use the CBCT postoperatively to avoid false density values about the augmented sinus, as it was to give the density of the bone graft material and not the newly formed bone around the implants. Besides, ethical obligation to avoid subjecting the patients to four CBCT examinations within a period of 6 months particularly if the immediate postoperative was not valid as a measurement tool. Thus, OPG was performed to decrease the $\mathrm{x}$-ray dose to the patients. 
The edentulous maxillary posterior region is a great challenge to rehabilitate with dental implants. This area has the poorest bone quality in the oral cavity with almost $50 \%$ D3 bone and 40\% D4. In D4 bone, the implant-bone contact is the least compared to higher bone densities. Bearing in mind the high masticatory stresses in the premolar molar area, the load directed to an implant in such weak bone will not be confined to the crest only but will likely extend farther toward the implant apex leading to bone resorption. Furthermore, the lack of cortical bone on the crest weakens the initial implant stability besides loss of bone volume rapidly after extraction due to maxillary sinus pneumatization and bone resorption. ${ }^{3,34}$ Such challenges necessitates the application of suitable osteotomy techniques, bone augmentation modalities, and appropriate reliable biomaterials to improve the bone quality, implant stability, and long term survival.

The primary implant stability is the outcome of several interacting factors. Those are the surgical osteotomy technique employed, bone density, and implant design and surface treatment. ${ }^{29,35}$ The osteotomy technique has an impact on both bone quality and quantity around dental impalnts. ${ }^{36}$ Bone density is indicative of bone quality and has been assessed in the current study by CBCT. Assessing the implant stability, in a quantified manner, in research had been tried using reverse torque testing. This method is invasive and can jeopardize the bone-implant interface and hence destroy osseointrgration. It could be only useful at the time of insertion but not later so it is not clinically reliable. ${ }^{29}$ The Periotest system designed to measure tooth mobility was used as a quantitative method to assess implant stability. However, this technique was found sensitive to a wide range of factors such as device tip placement angle, pillar height, and the metallic tip-implant distance. ${ }^{30}$ Meredith described the resonance frequency analysis or Osstell method as a noninvasive clinical modality to detect implant stability. ${ }^{37}$ Over the last decade, several generations of this device have been developed for implant stability measurement and had gained more popularity. ${ }^{28,29}$ However, the method involves a device and smart pegs to be attached to the tested implants. Those pegs also add to the cost, as they are not reusable or amenable to sterilization. The reproducibility and reliability of Osstell ISQ had been tested and well proven..$^{38}$ Thus, it was the device of choice in our study for monitoring stability.

Wallace and Forum performed a systematic review to investigate the effect of sinus augmentation on the survival of dental implants. According to that study, some authors advocated that the lateral osteotomy window of the maxillary sinus should be protected with a membrane (such as collagen membranes) to avoid what they called invagination of the mucogingival tissues. Moreover, they added that a logical explanation for such phenomenon was that the sinus cavity, after removal of the cortical lateral wall, must be protected with a barrier like a guided bone regeneration area. In a case series among the investigated, PRF membranes were utilized as the only external protection membrane for the sinuses. The radiographic follow up of such case series did not show any invagination. The results seemed to point out that PRF membranes were capable of protecting the sinus-augmented area. ${ }^{13}$ In our study, PRF membrane could not be placed in the control group to avoid violating its reliability as control with absence of PRF as the controlled variable. In the study group, we relied on presence of the PRF as an integral part of the graft.

Tarnow et al investigated bilateral sinus augmentation with or without barrier membrane in 12 patients. They concluded that barrier placement increased vital bone formation and had a positive effect on implant survival. They also recommended that barrier placement should be considered for all sinus elevations. ${ }^{39}$ Tawil and Mawla placed 61 implants in 30 sinuses augmented with Bio oss with or without concomitant use of collagen 
barrier (Bio-gide). They performed that via lateral osteotomy approach and followed up the patients for an average of 22.4 months. They concluded that membrane coverage of the osteotomy site led to improvement of the graft healing quality and the survival rate of the implants that were loaded 6 to 9 months after insertion. ${ }^{40}$ The current study methodology and findings are contradicting to the hypothesis of the necessity of using a barrier membrane over the lateral osteotomy. The use of membrane could have added to cost as well as adding another control variable. The clinical and radiographic follow up findings of our study had shown normal buccal cortical plate contour and no mucogingival invagination. Tawil and Mawla had placed more than one implant in each augmented sinus so this might be an indication for membrane barrier, when performing larger osteotomy. ${ }^{40} \mathrm{We}$ believe that single implant lateral sinus osteotomy does not necessitate placing a barrier membrane and if needed, PRF membrane would be sufficient.

Choukroun et al conducted a histologic assessment of the effect of adding PRF to freezedried bone allograft (FDBA) on sinus graft maturation. ${ }^{26}$ They concluded that adding PRF to FDBA in sinus augmentation led to healing time reduction prior to implant placement. Furthermore, from a histologic perspective, they added that healing time could be reduced to 4 months. Diss et al performed a pilot study in which they placed 35 implants in osteotome sinus elevation using PRF as the sole graft material. ${ }^{27}$ They followed up the cases for one year and the survival rate was $97.1 \%$ (1 implant loss). They concluded that PRF helped endosinus bone formation and after 1 year, new recognizable bone was verified radiographically. Furthermore, they found that a healing time of 2-3 months was sufficient to resist the torque applied during abutment tightening $(25 \mathrm{~N} . \mathrm{cm})$. The results of the current study were quite similar to these findings. The addition of PRF to the Bio oss bovine bone in sinus augmentation led to significantly higher values of implant stability as well as bone density at 6 months postoperatively. Accordingly, all the implants of study group were loaded after six months as they showed ISQ values more than 70. Whereas the loading of all the implants in the control group was delayed for up to ten months to reach the eligible ISQ. The survival rate after 10 months was $100 \%$. These findings were likely attributed to the ability of PRF to enhance the bone maturation.

Two cases in the study group had small tear of the membrane during the lifting procedure, PRF membrane was used to cover the tear successfully. The management of the tear with PRF was simple and economic. One case in the control group had small membrane tear and collagen membrane was used to manage it. The collagen membrane was more technique sensitive in use and was obviously more expensive. The PRF membrane may improve the healing of a Schneiderian membrane and stimulate its periosteum-like behavior and perhaps increase or stabilize the bone volume around the implant end. ${ }^{41-43}$

From the practical point of view, PRF addition to the Bio oss for sinus augmentation is cost effective via reducing the amount of xenograft used. Likewise, the use of PRF membrane on a Schneiderian membrane tear or as a barrier over the lateral osteotomy window is a very simple, economic, and biologic protection that can be used as a daily practice. Thus, it has been concluded that PRF addition to the sinus augmentation is more superior due to its bone maturation enhancing ability that resulted in higher implant stability and bone density and reduced loading time.

\section{ACKNOWLEDGMENTS}

The authors would like to thank the staff members and residents of oral and maxillofacial surgery and oral radiology departments for their help and effort throughout the study. 


\section{DISCLOSURE}

The authors declare clearly that there is no conflict of interest regarding the present study.

\section{REFERENCES}

1. Brånemark P I., Hansson B O, Adell R, Breine U, Lindström J, Hallén O, \& Ohman A. Osseointegrated implants in the treatment of the edentulous jaw. Experience from a 10-year period. Scand J Plast Reconstr Surg, 1977; 16: $1-132$.

2. Steigmann M, Garg AK. A comparative study of bilateral sinus lifts performed with platelet rich plasma alone versus alloplastic graft material reconstituted with blood. Implant Dent. 2005; 14:261-6. [PubMed: 16160572]

3. Misch CE. Bone density: A key determinant for treatment planning. In Misch CE, editor: Contemporary implant dentistry, St Louis, 2008, Mosby.

4. Winkler S. Implant site development and alveolar bone resorption patterns. J Oral Implantol 2002; 28:226e9.

5. Raja SV. Management of the posterior maxilla with sinus lift: Review of techniques. J Oral Maxillofac Surg 2009:67; 1730-4.

6. Toffler M. Minimally invasive sinus floor elevation procedures for simultaneous and staged implant placement. N Y State Dent J 2004; 70:38-44

7. Tong DC, Rioux K, Drangsholt M, Bierne OR. A review of survival rates for implants placed in grafted maxillary sinuses using meta-analysis. Int J Oral Maxillofac Impl 1998; 13: 175-182.

8. Misch CE: Maxillary sinus anatomy, pathology, and graft surgery In Misch CE, editor: Contemporary implant dentistry, St Louis, 2008, Mosby.

9. Tatum $\mathrm{OH}$ : Lecture presented at Alabama Implant Study Group, Birmingham, Ala, 1977.

10. Tatum $\mathrm{OH}$ : Maxillary and sinus implant reconstruction, Dent Clin North Am 1986; 30:107-119.

11. Misch CE: Maxillary sinus augmentation for endosteal implants: organized alternative treatment plans, Int J Oral Implantol. 1987; 4:49-58.

12. Summers RB. A new concept in maxillary implant surgery: The osteotome technique. Compendium. 1994; 15:152, 154-156, 158 passim; quiz 162.
13. Wallace SS, Froum SJ. Effect of maxillary sinus augmentation on the survival of endosseous dental implants. A systematic review. Ann Periodontol. 2003; 8: 328-343.

14. Daniel D, Rao SG. Evaluation of increase in bone height following maxillary sinus augmentation using direct and indirect technique. J Dent Implant. 2012;2:26-31

15. Pal US, Sharma NK, Singh RK, Mahammad S, Mehrotra D, Singh N, Mandhyan D. Direct vs. indirect sinus lift procedure: A comparison. Natl J Maxillofac Surg 2012; $3: 31-7$.

16. Nedir R, Bischof M, Vazquez L, Szmukler-Moncler S,Bernard JP. Osteotome sinus floor elevation without grafting material: A 1-year prospective pilot study with ITI implants. Clin Oral Implants Res. 2006;17:679-686

17. Browaeys H, Bouvry P, De Bruyn H. A literature review on biomaterials in sinus augmentation procedures. Clin Implant Dent Relat Res. 2007; 9:166-177

18. Jensen OT, Shulman LB, Block MS, et al. Report of the Sinus Consensus Conference of 1996. Int J Oral Maxillofac Implants. 1998; 13 suppl: 11-45.

19. Choukroun J, Adda F, Schoeffler C, et al. An opportunity in perio-implantology: The PRF [in french]. Implantodontie. 2001; 42:55-62.

20. Dohan Ehrenfest DM, de Peppo GM, Doglioli P, Sammartino G. Slow release of growth factors and thrombospondin-1 in Choukroun's platelet-rich fibrin (PRF): A gold standard to achieve for all surgical platelet concentrates technologies. Growth Factors. 2009; 27:63-69.

21. Choukroun J, Diss A, Simonpieri A, Girard MO, Schoeffler C, Dohan SL, Dohan AJ, Mouhyi J, Dohan DM. Plateletrich fibrin (PRF): A second generation platelet concentrate. Part IV: Clinical effects on tissue healing. Oral Surg Oral Med Oral Pathol Oral Radiol Endod. 2006; 101:e56-e60.

22. Charrier JB, Monteil JP, Albert S, Collon S, Bobin S, Dohan Ehrenfest DM. Relevance of Choukroun's platelet-rich fibrin (PRF) and SMAS flap in primary reconstruction after superficial or subtotal parotidectomy in patients with focal pleomorphic adenoma: A new technique. Rev Laryngol Otol Rhinol (Bord). 2008; 129: 313-318.

23. Simonpieri A, Dohan D. The relevance of PRF during complex bimaxillary rehabilitations, using multiple bone grafts, post-avulsion implantations and immediate loading: A case report [in french]. Implant. 2005; 11:33-47.

24. Saadoun AP, Touati B. Soft tissue recession around im- 
plants: Is it still unavoidable? - Part II. Pract Proced Aesthet Dent. 2007; 19:81-87; quiz 88.

25. Simonpieri A, Del Corso M, Sammartino G, Dohan Ehrenfest DM. The relevance of Choukroun's platelet-rich fibrin and metronidazole during complex maxillary rehabilitations using bone allograft. Part I: A new grafting protocol. Implant Dent. 2009; 18: 102-111.

26. Choukroun J, Diss A, Simonpieri A, Girard MO, Schoeffler C, Dohan SL, Dohan AJ, Mouhyi J, Dohan DM. Platelet-rich fibrin (PRF): A second-generation platelet concentrate. Part V: Histologic evaluations of PRF effects on bone allograft maturation in sinus lift. Oral Surg Oral Med Oral Pathol Oral Radiol Endod. 2006; 101:299-303.

27. Diss A, Dohan DM, Mouhyi J, Mahler P. Osteotome sinus floor elevation using Choukroun's platelet-rich fibrin as grafting material: A 1-year prospective pilot study with microthreaded implants. Oral Surg Oral Med Oral Pathol Oral Radiol Endod. 2008; 105:572-579.

28. Sennerby L, Meredith N. Implant stability measurements using resonance frequency analysis: biological and biomechanical aspects and clinical implications. Periodontol 2000. 2008; 47:51-66.

29. Herrero-Climent M, Santos-García R, Jaramillo-Santos R, Romero-Ruiz M M, Fernández-Palacin A, LázaroCalvo P, Bullón P, Ríos-Santos J V. Assessment of Osstell ISQ's reliability for implant stability measurement: A cross-sectional clinical study. Med Oral Patol Oral Cir Bucal. 2013; Nov; 18(6): e877-e882

30. Teerlinck J, Quirynen M, Darius P, van Steenberghe D. Periotest: an objective clinical diagnosis of bone apposition toward implants. Int J Oral Maxillofac Implants. 1991; Spring; 6(1):55-61.

31. Lundgren S, Cricchio G, Palma VC, Salata LA, Sennerby L. Sinus membrane elevation and simultaneous insertion of dental implants: A new surgical technique in maxillary sinus floor augmentation. Periodontol 2000. 2008; 47:193205.

32. Misch CE: Bone physiology, metabolism, and biomechanics. In Misch CE, editor: Contemporary implant dentistry, St Louis, 2008, Mosby.

33. Frost HM: The regional acceleratory phenomenon: a review. Henry Ford Hosp Med J. 1983; 31:3
34. Misch CE: Treatment planning for the edentulous posterior maxilla. In Misch CE, editor: Contemporary implant dentistry, St Louis, 2008, Mosby.

35. Glauser R, Sennerby L, Meredith N, Rée A, Lundgren A, Gottlow J, Hämmerle $\mathrm{CH}$. Resonance frequency analysis of implants subjected to immediate or early functional occlusal loading. Successful vs. failing implants. Clin Oral Implants Res. 2004 Aug; 15(4):428-34.

36. Abdelmabood A A, Abdelkarim M A, Hamed M S, Elsholkamy M A. Assessment of Bone Healing Around Immediately Loading Dental Implants in Posterior Maxilla with Two Different Osteotomy Techniques. Oral Med Oral Surg Oral Pathol Oral Radiol. 2015; 1(2): 66-76.

37. Meredith N. Assessment of implant stability as a prognostic determinant. Int J Prosthodont. 1998 Sep-Oct; 11(5):491-501.

38. Buyukguclu G, Ozkurt-Kayahan Z, Kazazoglu E. Reliability of the Osstell Implant Stability Quotient and Penguin Resonance Frequency Analysis to Evaluate Implant Stability. Implant Dentistry. 2018; 27(4):429-433

39. Tarnow DP, Wallace SS, Froum SJ, Rohrer MD, Cho SC. Histologic and clinical comparison of bilateral sinus floor elevations with and without barrier membrane placement in 12 patients: part 3 of an ongoing prospective study. Int $\mathrm{J}$ Periodontics Restorative Dent. 2000; 20:116-125.

40. Tawil G, Mawla M. Sinus floor elevation using a bovine bone mineral (Bio-Oss) with or without the concomitant use of a bilayered collagen barrier (Bio-Gide): A clinical report of immediate and delayed implant placement. Int J Oral Maxillofac Implants. 2001; 16: 713-721.

41. Dohan Ehrenfest DM, Diss A, Odin G, Doglioli P, Hippolyte MP, Charrier JB. In vitro effects of Choukroun's PRF (platelet-rich fibrin) on human gingival fibroblasts, dermal prekeratinocytes, preadipocytes, and maxillofacial osteoblasts in primary cultures. Oral Surg Oral Med Oral Pathol Oral Radiol Endod. 2009; 108:341-52.

42. Van Hinsbergh VW, Collen A, Koolwijk P. Role of fibrin matrix in angiogenesis. Ann N Y Acad Sci 2001; 936:426437.

43. Clark RA. Fibrin and wound healing. Ann N Y Acad Sci. 2001; 936:355-367. 